\title{
OPEN A stroke detection and discrimination framework using broadband microwave scattering on stochastic models with deep learning
}

\author{
Leeor Alon ${ }^{\bowtie}$ \&eena Dehkharghani
}

Stroke poses an immense public health burden and remains among the primary causes of death and disability worldwide. Emergent therapy is often precluded by late or indeterminate times of onset before initial clinical presentation. Rapid, mobile, safe and low-cost stroke detection technology remains a deeply unmet clinical need. Past studies have explored the use of microwave and other small form-factor strategies for rapid stroke detection; however, widespread clinical adoption remains unrealized. Here, we investigated the use of microwave scattering perturbations from ultra wide-band antenna arrays to learn dielectric signatures of disease. Two deep neural networks (DNNs) were used for: (1) stroke detection ("classification network"), and (2) characterization of the hemorrhage location and size ("discrimination network"). Dielectric signatures were learned on a simulated cohort of 666 hemorrhagic stroke and control subjects using 2D stochastic head models. The classification network yielded a stratified K-fold stroke detection accuracy $>94 \%$ with an AUC of 0.996, while the discrimination network resulted in a mean squared error of $<0.004 \mathrm{~cm}$ and $<0.02 \mathrm{~cm}$, for the stroke localization and size estimation, respectively. We report a novel approach to intelligent diagnostics using microwave wide-band scattering information thus circumventing conventional image-formation.

According to WHO estimates, stroke remains the second leading cause of death worldwide, and a leading cause for disability ${ }^{1}$. CT and MRI are central to stroke care; however, safety concerns relating respectively to ionizing radiation and the use of strong magnetic fields, as well as limits to portability and high costs, preclude their practical use in most pre- and non-clinical settings ${ }^{2,3}$. Further, profound disparities in the availability of such technologies perpetuate large imbalances in global health in the 21 st century, disproportionately affecting underserved communities, with the WHO estimating nearly two-thirds of the world's population as lacking access even to the most basic of medical imaging ${ }^{4}$. The consequence of delayed access is astronomical, with 1.9 million neurons, 14 billion synapses, and twelve kilometers of myelinated white matter lost every minute, hence the axiom, time is brain. Minimizing the time required for diagnosis and care for ischemic stroke is, therefore, paramount ${ }^{5}$. Rupture of cerebral blood vessels, producing hemorrhagic stroke, may be similarly catastrophic and necessitates prompt diagnosis ${ }^{6}$. In particular, subarachnoid hemorrhage, such as from rupture of cerebral aneurysms, is a potentially devastating injury occurring precipitously and often without warning in otherwise healthy individuals ${ }^{78}$; including the sizeable percentage of those succumbing even before hospital arrival, 30-day mortality rates in some series exceed $40 \%^{9,10}$. Such diseases thus demand fast, accurate, and safe characterization to facilitate diagnosis, prognostication, and treatment selection ${ }^{7,8}$.

Magnetic resonance imaging (MRI) devices have been explored in acute-care settings ${ }^{11}$, but wide scale dissemination to the pre-clinical environment is limited by cost, technical and logistical constraints (e.g. shielding, size, safety, etc.), and access in underserved populations ${ }^{12}$. Recently, several approaches for low-field $(<0.3 \mathrm{~T})$, low-cost, head MRI scanners have been proposed for acute-phase imaging (e.g. emergency departments, intensive care units, etc.). However, in the context of stroke detection, experience with such devices is preliminary and mobile deployment has yet to be fully realized ${ }^{13,14}$. Mobile computerized tomography $(\mathrm{CT})$ units have been 
used in acute settings, but remain encumbered by concerns associated with ionizing radiation ${ }^{15}$, low sensitivity for hyper-acute ischemic stroke without the addition of iodinated contrasts, cost, and portability ${ }^{16}$.

Other imaging approaches have been explored for stroke detection, including measurement of electrical properties (EP) of tissues using transcranial currents; however, dielectric map reconstruction is limited by the localization of induced currents to the tissue surfaces and the ill-posedness of the reconstruction ${ }^{17}$; consequently, deep-seated structures may not be unambiguously resolved with high fidelity, limiting anatomic and physiologic detail and clinical applicability ${ }^{18}$. Mapping of EP alternatively with radio-frequency waves has emerged as a potential diagnostic and/or imaging approach, leveraging the propagation characteristics of electromagnetic waves in optically opaque media ${ }^{18}$. Near-field microwave imaging (MI) systems estimate the spatial distribution of tissue EP by measuring the scattered field from an array of antennas, as initially described by Lin and Clark ${ }^{18}$ and subsequently by Semnov ${ }^{19,20}$ Presson $^{21}$, and Abbosh ${ }^{22}$.

MI methods can be divided into two broad categories: 1 . quantitative imaging of the dielectric properties of tissues (conductivity and relative permittivity) and 2. qualitative depiction of the geometrical features of the object as probed by the scattered fields ${ }^{23}$. Several potential solutions to the quantitative approach have been proposed according to physical models. These include the contrast source inversion (CSI) approach ${ }^{24}$, experimentally revealing low resolution images for high dielectric contrasts ${ }^{25}$, the linear inversion with truncated singular value decomposition (TSVD) and others ${ }^{26}$. However, the inherent non-linearity and ill-posedness of the inverse reconstruction problem remains challenging ${ }^{23}$, often necessitating regularization and/or parameter tuning. Finite Difference Time Domain (FDTD) electromagnetic field simulations have been used to ameliorate the MI reconstruction problem in which experimental data is compared against virtual simulations of the setup (solving Maxwell's equations in silico). Since the inverse problem is ill-posed, due to the degrees of freedom exceeding the number of measured values, Tikhonov regularization ${ }^{26}$ is applied. Modeling the architectural and dielectric complexity of the human brain present ongoing challenges ${ }^{23}$. By contrast, qualitative techniques, which aim to represent an image of the scattered field, have been proposed ${ }^{27-29}$, but lack information on the dielectric properties of tissues which may improve diagnostic value ${ }^{23}$.

Early work in MI hardware consisted of a vector network analyzer (VNA) connected to a computer control unit driving an antenna array. The forward and reflected power measured by each transmitting and receiving antenna is then used to calculate the S-parameter matrix, capturing the coupling between induced electromagnetic field and the head. Often a matching medium is used to improve energy transfer from the antennas to the head. Initial works utilized narrow-band directional antennas ${ }^{18}$ or open wave-guides ${ }^{20}$, often operating between 0.8 and $1.2 \mathrm{GHz}$. These frequencies were chosen due to the electromagnetic wave penetration and tissue dielectric contrast at these frequencies, enabling probing of changes in dielectric properties in deep-seated regions with ample spatial resolution ${ }^{23}$. More recently, wide-band systems (typically under $6 \mathrm{GHz}$ ) have become more mainstream, providing greater information about the electrical disturbances and obtained with larger frequency sweep $^{30-32}$. Software defined radio (SDR) technology has further advanced MI by reducing the cost of signal transmission and reception technology ${ }^{33}$.

The ongoing need for low cost, rapidly deployable, and scalable solutions for preclinical stroke diagnosis motivates the development of novel stroke detection methods. Here, we present a framework for hemorrhagic stroke detection using learned scattering signatures from ultra wide-band antenna arrays between 0.5 and 6 $\mathrm{GHz}$. While it is well established that changes in the dielectric properties of the brain are altered with disease, we investigated whether microwave scattering perturbations can be used to learn disease signatures. The potential to learn the dielectric signatures of strokes using big-data, on a population level, remains, to the best of our knowledge, unrealized. Herein, deep neural networks (DNNs) in combination with scattering information from microwave sources were used to classify and discriminate the presence of stroke. Results were shown for stochastic in silico, multi-tissue head models representing the dielectric disturbances occurring in the setting of hemorrhagic stroke and derived from electromagnetic field simulations. The results introduce a novel approach for intelligent diagnostics, circumventing conventional image-formation.

\section{Methods}

Electromagnetic (EM) field simulations. Simulations where conducted using the COMSOL finite element method (FEM) software (COMSOL Multiphysics, Burlington, MA USA) on an array of 8 wide-band horn antennas, each with an aperture of $120 \mathrm{~mm}$ and driven using a waveguide excitation. Each antenna was placed azimuthally around head models at 45 degree increments and filled with a dielectric with relative permittivity of 50 to reduce the cutoff frequency. The setup is summarized in Fig. 1A. A two-dimensional (2D) solver was used to reduce computational time as a large number of simulations were necessary for the varying anatomycoil-frequency conditions. Simulations were conducted by alternating with a single active port while recording the voltage on every port to calculate the S-parameter matrix at each frequency. A stationary frequency domain direct solver using the Newton method was used to ensure convergence. A total of 20 frequency sweeps were conducted at equal increments between 0.5 and $6 \mathrm{GHz}$ and were prescribed to inform DNNs trained for classification and discrimination of stroke. After the simulations concluded, random Gaussian noise with mean zero and standard deviation of $-100 \mathrm{dBm}$ was added to the S-parameters; this noise was introduced to represent the noise floor in commonly-used network analyzers ${ }^{34}$ such as can be incorporated in a system design to acquire S-parameters for stroke detection ${ }^{22}$. For each head simulation a complex matrix with dimension equal to: number of channels $\mathrm{x}$ number of channels $\mathrm{x}$ number of frequencies was obtained. EM field computations and post-processing were executed on a simulation workstation with a dual-core Xeon E5-2630 CPU with 128 GB of RAM.

In initial projections of feasibility for planned, future human subjects studies across our academic medical center, we abstracted an average of 600-700 eligible subjects over a span of 3 years, including allowances 
A

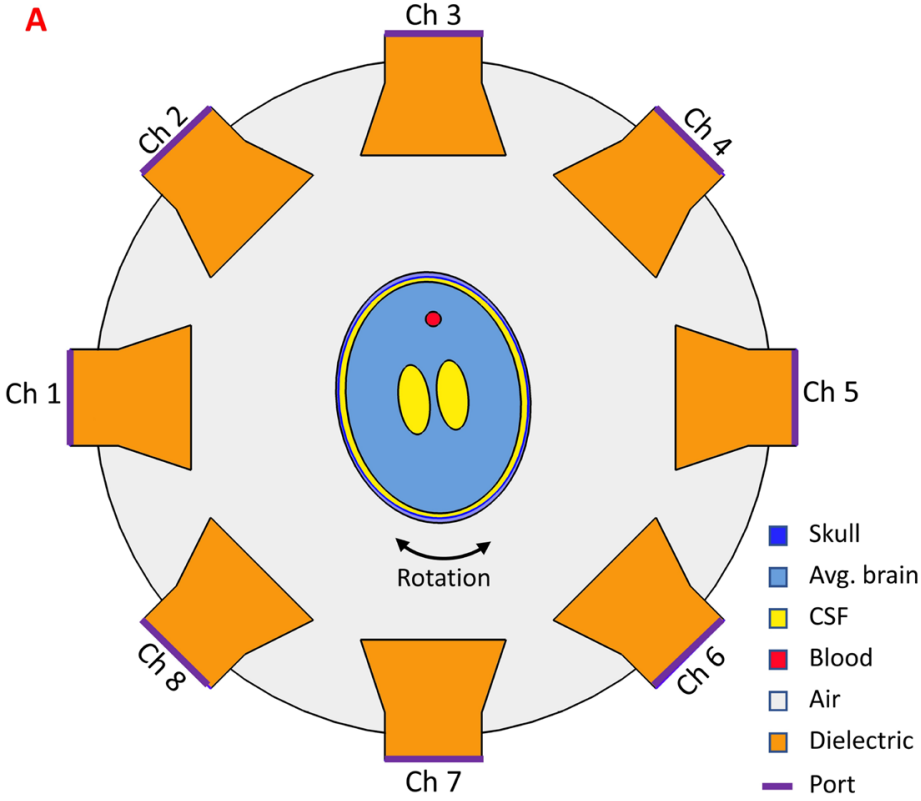



Figure 1. (A) Simulation setup for for FEM solver. 8-horn antennas were placed around varying 2D multitissue head models. (B) Flow chart for processing of EM field simulations, allowing modification of the head models, dielectric properties, and induction of stroke, simulating different frequencies and excitations.

\begin{tabular}{|l|l|l|l|r|r|l|}
\hline Anatomy & Orientation & Mean & Std. & Min & Max & Unit \\
\hline \multirow{2}{*}{ Head size } & x-direction & 20 & 2 & 13.8 & 25.6 & $\mathrm{~cm}$ \\
\cline { 2 - 8 } & $y$-direction & 26 & 2.6 & 19.8 & 33.0 & $\mathrm{~cm}$ \\
\hline \multirow{2}{*}{ Vent size } & $\mathrm{x}$-direction & 4.55 & 0.5 & 5.9 & 3.5 & $\mathrm{~cm}$ \\
\cline { 2 - 8 } & $y$-direction & 8.45 & 0.85 & 10.8 & 6.34 & $\mathrm{~cm}$ \\
\hline \multirow{2}{*}{ Head rot. } & xy-plane & 0 & 3.5 & -10.76 & 10.18 & deg. \\
\hline \multirow{2}{*}{ Blood size } & x-direction & 2 & 0.5 & 0.84 & 3.8 & $\mathrm{~cm}$ \\
\cline { 2 - 7 } & $y$-direction & 2 & 0.5 & 0.84 & 3.8 & $\mathrm{~cm}$ \\
\hline
\end{tabular}

Table 1. Head anatomy variation.

for control subjects. Our initial simulation, therefore, aimed to reasonably recapitulate this projection, and computations were initiated accordingly. The simulation demanded approximately one month of continuous computation to achieve the predetermined scale of simulations $(>100,000)$. We elected to analyze the results after the initial month of computation, which converged at the milestone of 666 subjects, all of which were used in the experimentation below.

Head EP perturbation. All 666 head models were generated in simulation using COMSOL Livelink, enabling scripting within the Matlab (Mathworks, Natick, Massachusetts USA) environment. A 2D synthetic, transaxial slice through the brain, was modelled based on the 3D Specific Anthropomorphic Mannequin (SAM) mode ${ }^{35}$, producing a simulated central slice through the brain at the level of the lateral ventricles. Stochastic modifications to the brain dimensions were applied to each head model, comprising variation in the width and length of the head and brain independently, and width and length of the lateral ventricles. The head models were placed centrally within the antenna array, with random reorientation of the head prescribed relative to the array, in order to represent varying head orientations occurring when the head is fixated to a plastic stereotactic frame permitted to pivot relative to the antenna array, yet remains immobile with respect to translation. Variations in head anatomy and rotation are summarized in Table 1. A total of five different tissues were included in the simulations: skull, blood, cerebrospinal fluid (CSF), average brain, and air. For each frequency of interest between 0.5 and $6 \mathrm{GHz}$, the dielectric properties were extracted from Gabriel et al. ${ }^{36-38}$. Random-valued head anatomies were fed to the DNN representing subject-wise variation of the head, thus presenting the DNN with highly varying conditions that disrupt the S-parameter matrices to facilitate learning. Fifty percent of the heads were selected at random and intracranial blood was introduced in random locations, simulating intraparenchymal hemorrhagic strokes. Sizes of the strokes were independently varied in the $\mathrm{x}$ - and $\mathrm{y}$-directions with a 2 $\pm 0.5 \mathrm{~cm}$ (mean \pm standard deviation) in diameter (ranging between 0.5 and $4 \mathrm{~cm}$, in the $\mathrm{x}$ - and $\mathrm{y}$-directions, 


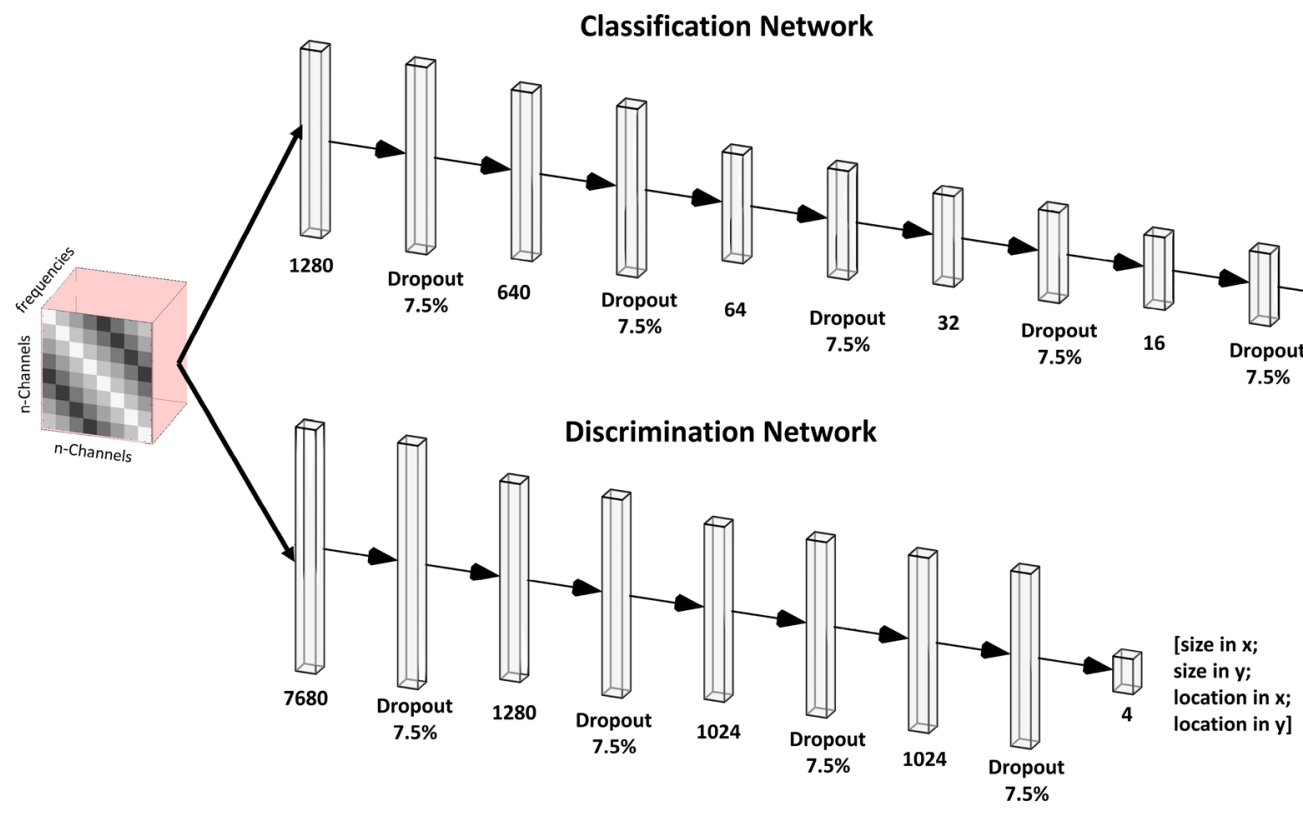

Figure 2. DNN architectures used for the classification and discrimination networks. Amplitude and phase of the multi-frequency S-parameter matrices were concatenated, vectorized and fed into each DNN. A sigmoid output and 4-vector linear output were used for the classification and discrimination networks, respectively.

independently) (Table 1). The stroke $\mathrm{x}$ - and $\mathrm{y}$-locations were selected from a uniform random distribution, with the outer bounds of the distribution changing depending on the size of the brain. A total of 106,560 simulations were conducted using different head anatomies, frequency sweeps, and antennas driven. Execution of these simulations was performed in $>720$ continuous hours of simulation time. The pipeline for stochastic head model simulations and computation of the S-parameter matrices at each frequency of operation is summarized in Fig. 1B. The effect of perturbations on the dielectric properties of the head induced by different strokes was analyzed and plotted across different frequencies.

Classification and discrimination. Multi-frequency S-parameter matrices were exported into python. The dimensions of each complex-valued matrix were $\mathrm{n}_{c h} \mathrm{x}_{c h} \mathrm{x}_{f}$, where $\mathrm{n}_{c h}$ is the number of channels and $\mathrm{n}_{f}$ is the number of simulated frequencies. Amplitude and phase of the complex-valued matrices were reformatted, for each model, to $\mathrm{n}_{\mathrm{ch}}{ }^{2}{ }^{\star} \mathrm{n}_{f}$. Training, testing, and validation of the neural net models were conducted in the Keras environment ${ }^{39}$. Before training, the data across subjects were split such that $70 \%$ of the data were used for training, $15 \%$ for validation, and $15 \%$ for testing, respectively. Training, testing, and validation data sets were normalized using a standard scalar normalizer such that the data was scaled to unit variance. Two distinct DNNs were used for the stroke diagnosis: the first DNN was used to classify the presence of hemorrhagic stroke using S-parameter matrix data ("Classification DNN") and the second DNN was used to discriminate the stroke location and size inside the head ("Discrimination DNN"). The classification DNN was trained using 666 stroke and control head models, consisting of 307 simulated strokes (46\%). The discrimination DNN was trained using the stroke-only cohort. A graphical depiction of the classification DNN comprising fully-connected layers followed by a final sigmoid activation layer is shown in Fig. 2, top. Input to the classification network comprised 1280 unique parameters (generated from the complex s-parameter matrices) and an input layer for the classification network accordingly set with the purpose of identifying frequency dependent coil-coupling relationships that are unique for identification of stroke. The discrimination DNN comprising a wider fully-connected architecture terminating in a 4-dimensional linear layer with the 4-dimensional vector representing the $\mathrm{x}$-, $\mathrm{y}$-locations and size of hemorrhage in the $\mathrm{x}$ - and $\mathrm{y}$-directions, respectively, thus characterizing the dimension and location of the hemorrhage 2, bottom. For both the classification and discrimination networks a dropout layer with dropout threshold of 7.5\% was introduced between the fully connected layers to reduce over-fitting. Each layer of the classification network was initialized using a truncated normal distribution with zero mean and standard deviation of 0.75 and the discrimination network was initialized using a truncated normal distribution with zero mean and standard deviation of 0.05. A summary of network details can be found in Supplemental Materials Tables 1 and 2. Model optimization for both networks was performed using the Adam optimizer ${ }^{40}$. A binary cross-entropy loss function ${ }^{41}$ with 20 epochs and a batch size of 100 was used for the classification network training, while a mean square error (MSE) loss function, 1000 epochs, and a batch size of 100 were used for discrimination network training. The loss function and accuracy of each trained network as a function of epoch was plotted. Classification accuracy was quantified using the stratified K-fold method ${ }^{42}$ with 10 splits. A Receiver-Operating Characteristic (ROC) curve was plotted and the Area Under the Curve (AUC) was computed. Sensitivity (recall), specificity, precision (positive predictive value), overall accuracy, and f1-scores and associated confidence intervals were computed for the classification network. The discrimination network was evaluated by calculation of the test-set MSE for the errors in predicted $\mathrm{x}-, \mathrm{y}$-locations and $\mathrm{x}-$, $\mathrm{y}$-stroke size. Further, object-detection accu- 
A

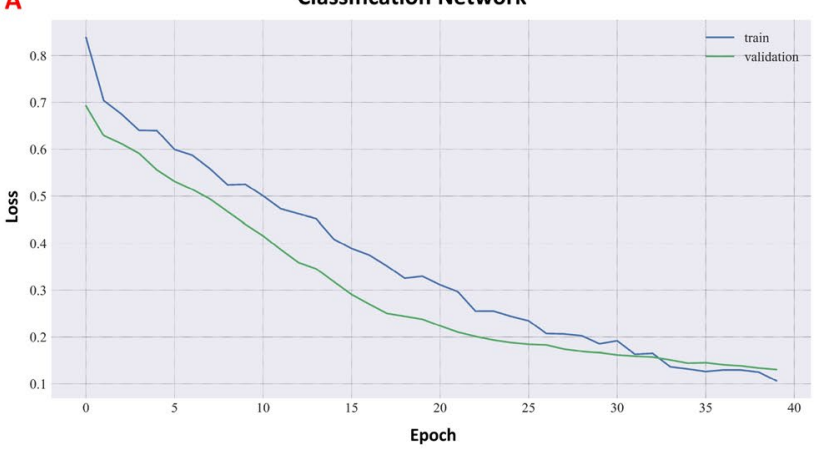

B

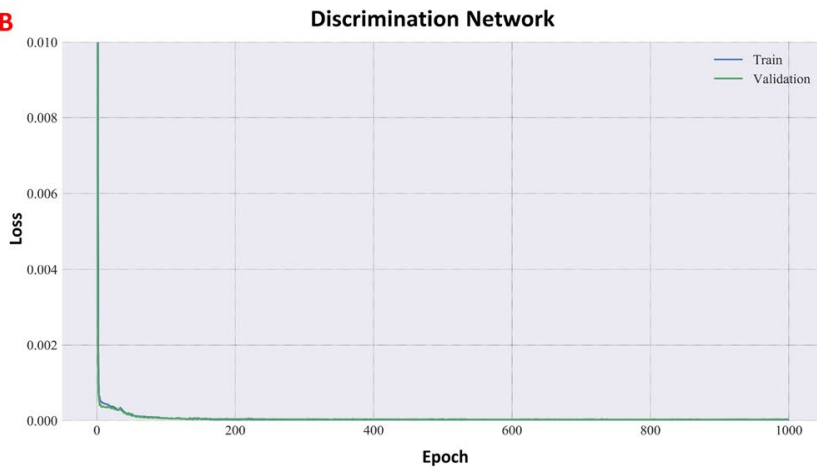

Figure 3. Classification network loss as a function of epoch number for the training and validation sets (A) and Training Discrimination network MSE as a function of epoch number for the stroke-only train and validation sets.
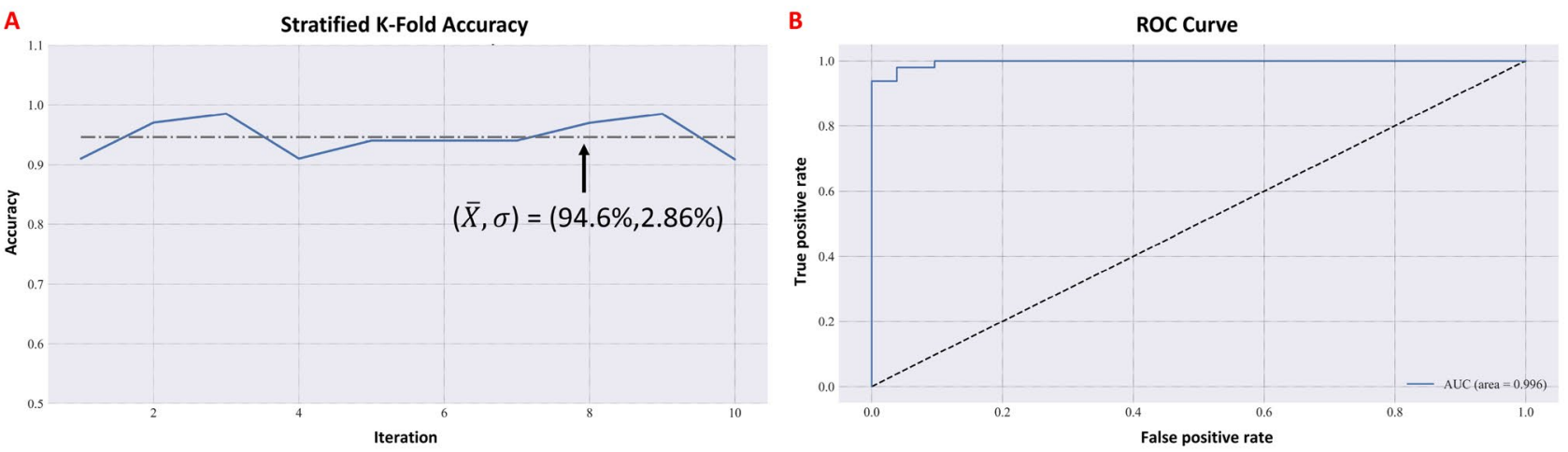

Figure 4. Stratified K-fold accuracy as a function of iteration for the classification network (blue); mean and standard deviation shown in black (A) and ROC curve computed using the test set predicted stroke classifications (B).

racy was evaluated using the mean and median intersection-over-the-union (IoU). For visualization purposes, results were compared against the ground truth in randomly-chosen stroke models selected from the test-set. Frequency- and antenna- dependent stroke dielectric effects were assessed by plotting changes in the magnitude of the S-parameter matrices occurring due to the presence of stroke for $0.5,1$ and $3 \mathrm{GHz}$ and presented $\mathrm{dB}$ scale.

\section{Results}

Classification. Execution of the classification network training was performed and the binary cross-entropy loss function is shown in Fig. 3A, demonstrating convergence of the network. Similarly, convergence of the discrimination network using the MSE loss function is shown in Fig. 3B. Generalization was evaluated using the stratified K-fold method ${ }^{42}$, exhibiting a mean model accuracy of $94.6 \%$ with a standard deviation of $\pm 2.86 \%$ (Fig. 4A). Test set ROC curve is shown in Fig. 4B, demonstrating an AUC of 0.996 for the detection of stroke, suggesting that the classification DNN generalizes well using broadband S-parameter measurements as inputs. Upon convergence of the classification network, test set prediction resulted in sensitivity $=0.89$ (95\% CI $0.83-$ $0.95)$, precision $=1.0$, specificity $=1.0$, accuracy $=0.94(95 \%$ CI $0.89-0.99)$, and f1-score $=0.94$.

Discrimination. The discrimination network was designed to predict both the size and location of the stroke; discrimination was performed via learning patterns from frequency dependent S-parameters from different antenna elements, rather than relying on conventional microwave imaging reconstruction. In order to better discriminate stroke spatial characteristics, a larger network with more redundancy was required, consisting of a $6 \times$ larger first layer, compared to the classification network, and a relatively larger number of neurons in the subsequent hidden layers, permitting finer prediction of size and location (Fig. 2, bottom). The discrimination network MSE as a function of epoch is shown in Fig. 3B, demonstrating close agreement between the test and validation set errors. Upon convergence of training, size and location of the strokes in 10 random head models from the test set cohort were plotted in Fig. 5, where the dotted red ovals represent the in silico stroke. As shown, varying head sizes as well as stroke locations and sizes were were used as inputs to the DNN. For clarity of representation, additional anatomic features (other than the stroke) were excluded. The predicted stroke size and location predicted by the DNN is illustrated as a $2 \mathrm{D}$ Gaussian probability density function shown in blue. Test set MSE for stroke localization in the $\mathrm{x}$ - and $\mathrm{y}$-directions was $0.0023 \mathrm{~cm}$ and $0.0043 \mathrm{~cm}$, respectively, and stroke 


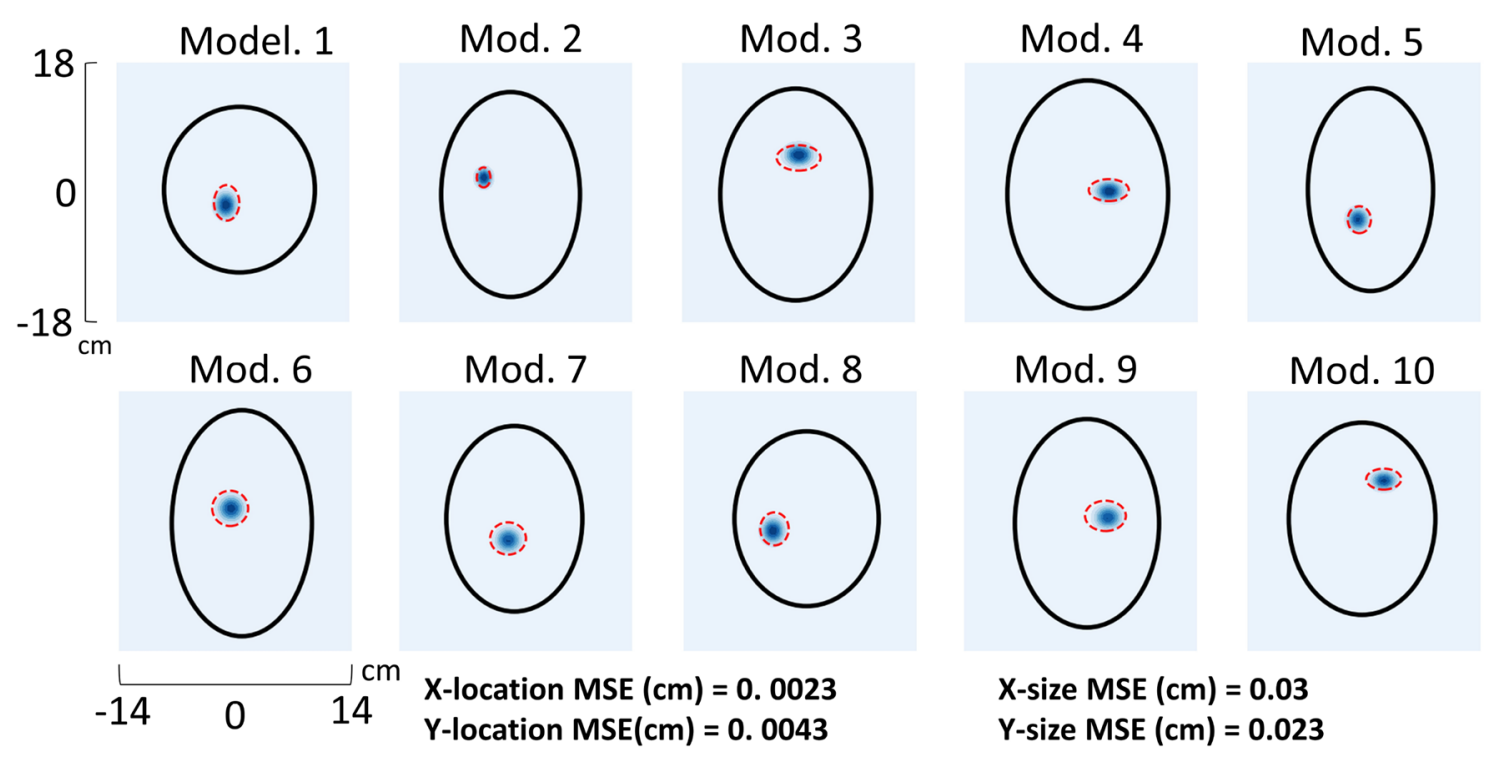

Figure 5. Test set stroke predicted by the discrimination network. True stroke size and location(red dotted line) is compared against the predicted strokes (blue Gaussians).

A

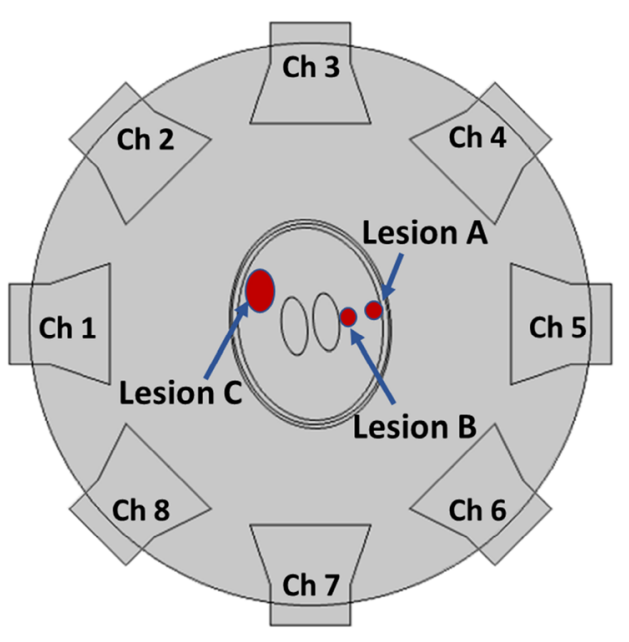

B
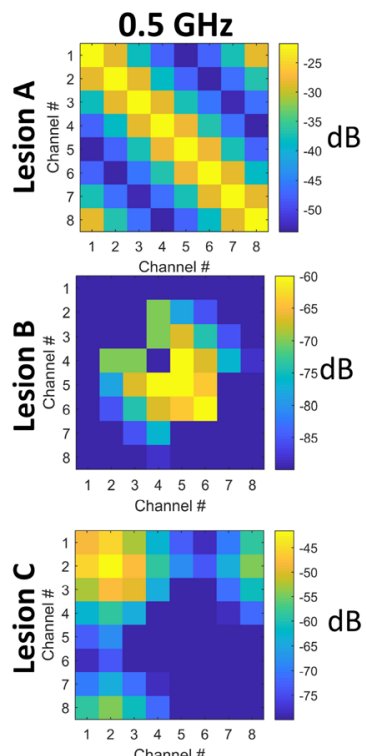
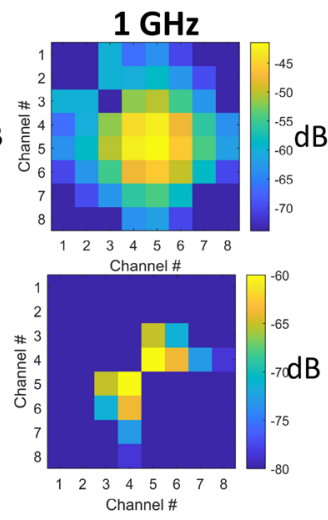

Channel \#

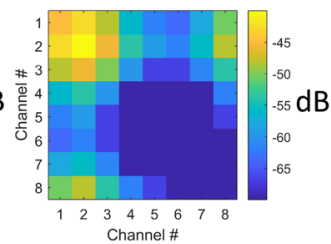

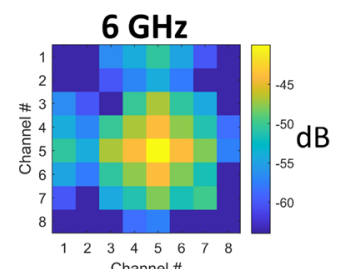
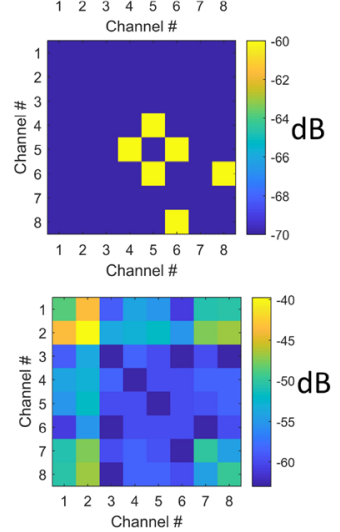

Figure 6. Frequency- and space-dependent changes in S-parameter values for different lesions.

size MSE in the $\mathrm{x}$ - and y-directions was $0.03 \mathrm{~cm}$ and $0.023 \mathrm{~cm}$, respectively. Results of object detection accuracy provided by mean and median IoU were 0.84 and 0.94 , respectively.

Frequency and antenna dependent dielectric effects. The observed changes in the S-parameters for three different strokes (represented as red ovals A, B and C) are summarized in Fig. 6 for different frequencies. Lesion A exhibited prominent changes in the S-parameters adjacent to antennas 4 and 5 occurring above 0.5 GHz. The change in S45 represents the increase in power transfer between antennas 4 and 5 occurring due to the changes in dielectric parameters in the brain induced by the presence of stroke. When the same-sized lesion (B), is displaced to an deeper region of the brain, changes in the S-parameters of channels 4, 5 and 6 are observed at the $0.5 \mathrm{GHz}$, as well as at higher frequencies. A larger lesion $\mathrm{C}$, in the contralateral side (relative to strokes $\mathrm{A}$ and B), changed the S-parameter values for channels 1 and 2 (closest to lesion C) and their respective coupling.

\section{Discussion}

In this work we demonstrated that physical changes in tissue EPs caused by hemorrhagic stroke cause measurable changes in EM wave scattering. These changes in the S-parameter matrices can be used to train networks for intelligent diagnosis and discrimination of stroke. Past studies have utilized narrow-band frequency excitation 
and reception systems ${ }^{19,43,44}$ motivated by the reported advantages of dielectric contrast in the brain at those frequencies; here, broadband acquisitions between 0.5 and $6 \mathrm{GHz}$ provided complementary information. Specifically, the importance of multi-antenna and multi-frequency information as was depicted in Fig. 6 underscored that higher S-parameter disruptions at higher frequencies occur due to the higher sensitivity in shallow depths, due to the skin effect, as well as the size of the lesion. These changes provided valuable information for the DNNs to facilitate learning the presence of stroke as well as the capability to localize and estimate the size of the stroke. In this respect, this study presents a first-of-its-kind approach whereby the additional contrast arising from a broadband frequency sweep in a simulated cohort could be utilized to facilitate learning and generalization, while circumventing conventional MI reconstruction inverse problems.

Two-dimensional EM simulations were employed due to computational demands inherent to three-dimensional simulations. Here, more than 100,000 simulations were conducted in order to calculate electromagnetic fields and thus S-parameters across all frequency, subject, and antenna permutations, requiring approximately 1 month of continuous computation. The challenges inherent to engineering realistic, 3D patho-anatomic models for electromagnetic simulation are well-recognized and constitute ongoing motivation for the development of robust experimental methods ${ }^{45}$. Reduction of the dimensionality to two-dimensions to alleviate computational demand consequently eliminated the z-dimension (i.e. the craniocaudal direction) thus converting the hemorrhage to an affected 2D area of the simulated brain with Maxwell's equations solved in 2D. Notwithstanding, we anticipate that a transition to three dimensions in realizable antenna array systems would benefit from the utilization of a greater number of antennas, thus capturing 3D spatial detail such as that arising from a 3D hemorrhage volume within the head. While past studies have demonstrated that large head-encircling arrays ( $>30$ antennas) together with electromagnetic field simulations can be used for stroke detection ${ }^{46,47}$, the benefits of machine learning approaches applied to large cohorts, including with broadband frequency interrogation, remain untested.

In this study we explore new avenues for machine learning-assisted MI, leveraging the additional contrast arising from broadband microwave frequencies, in order to improve prediction of the presence, location, and size of intracranial hemorrhage. Importantly, however, the optimal antenna configuration and prescription of interrogated frequencies requires further study to balance sensitivity with hardware and computational demand. We anticipate that tolerance to reductions will most likely be hardware- and application-specific and a topic for future study. The reduction in the number of frequency sweeps and/or coils used will result in acceleration of S-parameter acquisitions to improve patient comfort or time to diagnosis. Further, our selection of a simulated cohort of 666 stroke and control subjects constitutes only an initial approximation of an achievable cohort in contemporary stroke trials and is representative of stroke imaging workflow in our own practice.

Acquisition systems capable of realizing this approach could potentially employ wide-band antenna arrays encircling the patient's head. Each antenna can be connected to controllable RF/microwave switches routing each antenna to receive or transmit signals and connected to a multi-port vector network analyzer (VNA). Alternatively, SDRs can be utilized alongside directional couplers to gather wide-band scattering information. We expect that such systems will be two orders of magnitude less expensive than state of the art imaging systems (e.g. clinical MRI).

We elected to simulate intracerebral hemorrhage in this initial investigation. Blood provides a stronger dielectric contrast than ischemia, which may be more pathologically variegated at the tissue level, challenging development of realistic simulated conditions. While this study did not attempt to classify or discriminate ischemic stroke, the detection accuracy for hemorrhage $>1 \mathrm{~cm}$ in diameter (see Table 1 for stroke variation in $\mathrm{x}$ - and y-directions) was $94.6 \%$, and therefore the dependable exclusion of hemorrhage can serve as a valuable screening tool in the acute stroke setting to facilitate rapid thrombolysis as per current guidelines ${ }^{48}$ in early-presenting patients. This latter point bears emphasis, as intravenous thrombolysis remains the only approved pharmacologic therapy in acute ischemic stroke patients, but with rare exceptions cannot be administered beyond the initial, hyperacute window. Consequently, only $12 \%$ of patients receive critical intravenous thrombolysis, despite a $30 \%$ improvement in outcomes among those treated. Future studies will, nevertheless, focus on the detection of ischemic stroke as well as other neurologic disorders using the dielectrographic technique described here.

Overall, we present a method for hemorrhagic stroke detection and discrimination by means of learning disease dielectric signatures from S-parameter measurements using wide-band antenna arrays. We investigated whether microwave scattering perturbations can be used to learn disease signatures from a large simulated cohort. Two DNN architectures were designed and tuned for classification and discrimination in presence of stroke in stochastic in silico, multi-compartment head models representing the tissue dielectric disturbances occurring in the setting of hemorrhagic stroke and derived from electromagnetic field simulations. The nature of our proposed approach provides an effective potential strategy for rapid stroke detection and management, circumventing conventional image generation.

Received: 10 June 2021; Accepted: 22 November 2021

Published online: 20 December 2021

\section{References}

1. World Health Organisation. Global Status Report on Noncommunicable Diseases 2014, Technical Report (World Health Organisation, 2014).

2. Giroud, M., Jacquin, A. \& Béjot, Y. The worldwide landscape of stroke in the 21st century. The Lancet 383(9913), 195 (2014).

3. Mollura, D. J. \& Lungren, M. P. Radiology in Global Health: Strategies, Implementation, and Applications (Springer, 2014).

4. Ferns, S. P. et al. De novo aneurysm formation and growth of untreated aneurysms: A 5-year mra follow-up in a large cohort of patients with coiled aneurysms and review of the literature. Stroke 42, 313 (2011).

5. Saver, J. L. Time is brain-Quantified. Stroke 37, 263 (2006). 
6. Claude Hemphill, J. et al. Guidelines for the management of spontaneous intracerebral hemorrhage. Stroke 46, 2032 (2015).

7. Lantigua, H. et al. Subarachnoid hemorrhage: Who dies, and why? Crit. Care 19, 309 (2015).

8. Dority, J. S. \& Oldham, J. S. Subarachnoid hemorrhage: An update. Anesthesiol. Clin. 34, 577 (2016).

9. Chalouhi, N., Hoh, B. L. \& Hasan, D. Review of cerebral aneurysm formation, growth, and rupture. Stroke 44, 3613 (2013).

10. Skodvin, T. Ø., Johnsen, L.-H., Gjertsen, Ø., Isaksen, J. G. \& Sorteberg, A. Cerebral aneurysm morphology before and after rupture. Stroke 48, 880 (2017).

11. Sheth, K. N. et al. Assessment of brain injury using portable, low-field magnetic resonance imaging at the bedside of critically ill patients. JAMA Neurol. 02114(1), E1-E7 (2020).

12. Geethanath, S. \& Thomas Vaughan, J. Accessible magnetic resonance imaging: A review. J. Magn. Reson. Imaging 49(7), e65-e77 (2019).

13. Wald, L. L., McDaniel, P. C., Witzel, T., Stockmann, J. P. \& Cooley, C. Z. Low-cost and portable MRI. J. Magn. Reson. Imaging 52(3), 686-696 (2020).

14. Cooley, C. Z. et al. A portable scanner for magnetic resonance imaging of the brain. Nat. Biomed. Eng. 5, 229 (2020).

15. Miglioretti, D. L. et al. Pediatric computed tomography and associated radiation exposure and estimated cancer risk. JAMA Pediatr. 167(8), 700-707 (2013).

16. Fassbender, K. et al. Mobile stroke units for prehospital thrombolysis, triage, and beyond: Benefits and challenges. Lancet Neurol. 16(3), 227-237 (2017).

17. Brown, B. H. Electrical impedance tomography (EIT): A review. J. Med. Eng. Technol. 27(3), 972003 (2003).

18. Lin, J. C. \& Clarke, M. J. Microwave imaging of cerebral edema. Proc. IEEE 70(5), 523-524 (1982).

19. Semenov, S. Y. et al.. Microwave-tomographic imaging of the high dielectric-contrast objects using different image-reconstruction approaches. In IEEE Transactions on Microwave Theory and Techniques (2005).

20. Semenov, S. Y. \& Corfield, D. R. Microwave tomography for brain imaging: Feasibility assessment for stroke detection. Int. J. Antennas Propag. 2008, 254830 (2008).

21. Trefná, H. \& Persson, M. Antenna array design for brain monitoring. In IEEE International Symposium on Antennas and Propagation and USNC/URSI National Radio Science Meeting. APSURS, Vol. I (2008).

22. Mustafa, S., Mohammed, B. \& Abbosh, A. Novel preprocessing techniques for accurate microwave imaging of human brain. In IEEE Antennas and Wireless Propagation Letters (2013).

23. Crocco, L., Karanasiou, I., James, M. L. \& Conceição, R. C. Emerging Electromagnetic Technologies for Brain Diseases Diagnostics, Monitoring and Therapy (Springer, 2018).

24. Van Den Berg, P. M. \& Kleinman, R. E. A contrast source inversion method. Inverse Prob. 13(6), 1607-1620 (1997).

25. Abubakar, A., Van Den Berg, P. M. \& Mallorqui, J. J. Imaging of biomedical data using a multiplicative regularized contrast source inversion method. IEEE Trans. Microw. Theory Tech. 50(7), 1761-1771 (2002).

26. Bertero, M. \& Boccacci, P. Introduction to Inverse Problems in Imaging (CRC Press, 1998).

27. Colton, D. \& Kirsch, A. A simple method for solving inverse scattering problems in the resonance region. Inverse Prob. 12(4), 383-393 (1996).

28. Kirsch, A. Characterization of the shape of a scattering obstacle using the spectral data of the far field operator. Inverse Prob. 14(6), 1489-1512 (1998).

29. Catapano, I., Crocco, L. \& Isernia, T. On simple methods for shape reconstruction of unknown scatterers. IEEE Trans. Antennas Propag. 55(5), 1431-1436 (2007).

30. Jamlos. M. A., Jamlos, M. F. \& Ismail, A. H. High performance novel UWB array antenna for brain tumor detection via scattering parameters in microwave imaging simulation system. In 2015 9th European Conference on Antennas and Propagation, EuCAP 2015, 3-7 (2015).

31. Fei, C. et al. Ultrahigh frequency $(100 \mathrm{MHz}-300 \mathrm{MHz})$ ultrasonic transducers for optical resolution medical imagining. Sci. Rep. 6, 1-8 (2016).

32. Alqadami, A. S. M., Bialkowski, K. S., Mobashsher, A. T. \& Abbosh, A. M. Wearable electromagnetic head imaging system using flexible wideband antenna array based on polymer technology for brain stroke diagnosis. IEEE Trans. Biomed. Circuits Syst. 13(1), 124-134 (2019).

33. Stancombe, A. E., Bialkowski, K. S. \& Abbosh, A. M. Portable microwave head imaging system using software-defined radio and switching network. IEEE J. Electromagn. RF Microw. Med. Biol. 3(4), 284-291 (2019).

34. Agilnet. Understanding and Improving Network Analyzer Dynamic Range. Technical Report (Agilnet, 2000).

35. Gordon, C. C. et al. Anthropometric survey Of U.S. army personnel, summary statistics, interim report. NATICK/TR-89/027 (1988).

36. Gabriel, S., Lau, R. W. \& Gabriel, C. The dielectric properties of biological tissues: III. Parametric models for the dielectric spectrum of tissues. Phys. Med. Biol. 4, 2271 (1996).

37. Gabriel, C., Gabriel, S. \& Corthout, E. The dielectric properties of biological tissues: I. Literature survey. Phys. Med. Biol. 41, 2231 (1996).

38. Gabriel, S., Lau, R. W. \& Gabriel, C. The dielectric properties of biological tissues: II. Measurements in the frequency range $10 \mathrm{~Hz}$ to $20 \mathrm{GHz}$. Phys. Med. Biol. 41, 2251 (1996).

39. Chollet, F. Keras: Deep Learning Library for Theano and TensorFlow (2015).

40. Kingma, D. P. \& Ba, J. L. Adam: A method for stochastic optimization. In 3rd International Conference on Learning Representations, ICLR 2015-Conference Track Proceedings (2015).

41. Goodfellow, A. C. I. Yoshua Bengio (Deep Learning Book, 2015).

42. Salzberg, S. L. On comparing classifiers: Pitfalls to avoid and a recommended approach. Data Mining Knowl. Discov. 1, 137 (1997).

43. Abubakar, A., Habashy, T. M., Bloemenkamp, R. F. \& Berg, D. Solving Electromagnetic Inverse, Cross-correlated Contrast Source, and Inversion Method. Related Content A Contrast Source Inversion Method. Inverse Prob. 13, 1607-1620 (1997).

44. Mojabi, P., Ostadrahimi, M., Shafai, L. \& LoVetri, J. Microwave tomography techniques and algorithms: A review. In 2012 15th International Symposium on Antenna Technology and Applied Electromagnetics (2012).

45. George, A. Nested dissection of a regular finite element mesh. SIAM J. Numer. Anal. 10, 345 (1973).

46. Semenov, S. Microwave tomography: Review of the progress towards clinical applications. Philos. Trans. R. Soc. A Math. Phys. Eng. Sci. 367(1900), 3021-3042 (2009).

47. Semenov, S., Portnov, Y., Semenov, A., Korotkevich, A. \& Kokov, A. Neuroimaging patterns of cerebral hyperperfusion. J. Phys. Conf. Ser. https://doi.org/10.1088/1742-6596/886/1/012014 (2017).

48. Guidelines for the early management of patients with acute ischemic stroke: 2019 update to the 2018 guidelines for the early management of acute ischemic stroke a guideline for healthcare professionals from the American Heart Association/American Stroke Association (2019).

\section{Author contributions}

L.A. and S.D. wrote the manuscript text and designed the study. L.A. prepared the figures. All authors reviewed the manuscript. 


\section{Competing interests}

The authors declare no competing interests.

\section{Additional information}

Supplementary Information The online version contains supplementary material available at https://doi.org/ 10.1038/s41598-021-03043-y.

Correspondence and requests for materials should be addressed to L.A.

Reprints and permissions information is available at www.nature.com/reprints.

Publisher's note Springer Nature remains neutral with regard to jurisdictional claims in published maps and institutional affiliations.

(c) (1) Open Access This article is licensed under a Creative Commons Attribution 4.0 International License, which permits use, sharing, adaptation, distribution and reproduction in any medium or format, as long as you give appropriate credit to the original author(s) and the source, provide a link to the Creative Commons licence, and indicate if changes were made. The images or other third party material in this article are included in the article's Creative Commons licence, unless indicated otherwise in a credit line to the material. If material is not included in the article's Creative Commons licence and your intended use is not permitted by statutory regulation or exceeds the permitted use, you will need to obtain permission directly from the copyright holder. To view a copy of this licence, visit http://creativecommons.org/licenses/by/4.0/.

(C) The Author(s) 2021 